\title{
Regeneración ósea con fibrina rica en plaquetas e injerto óseo autólogo post extracción de canino impactado. Reporte de caso
}

\author{
Bone regeneration with platelet-rich fibrin and autologous bone graft after \\ extracting impacted canine. Case report
}

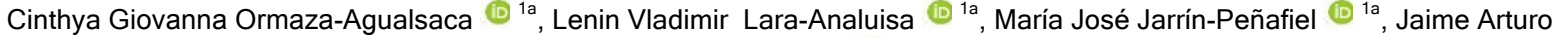 \\ RESUMEN \\ CastroPallaroso 10
}

El objetivo de este reporte de caso, es valorar el uso del injerto óseo autólogo y fibrina rica en plaquetas (FRP), como regeneración ósea guiada, después de la extracción de un canino retenido. Paciente femenino de 42 años, con antecedente de hipertensión controlada, acudió a la clínica odontológica SERODU (Ecuador), por presentar una prominencia en la región nasogeniana izquierda. Se le realizó una Tomografía Axial Computarizada (TAC), en la cual se observó a la pieza 1.3 incluida en el maxilar, en posición horizontal, la corona hacia mesial y la raíz hacia distal. Se decidió realizar la extracción del canino retenido y la colocación de biomateriales de regeneración a base de FRP e injerto de hueso autólogo, obtenido de la tuberosidad maxilar derecha. Se realizaron controles clínicos y radiográficos posteriores a la cirugía. Seis meses después se observó una cicatrización adecuada e imágenes compatibles con regeneración ósea en la zona intervenida.

Palabras clave: Diente impactado; Fibrina Rica en Plaquetas; Regeneración ósea; Tomografía computarizada; Tomografía Axial Computarizada (TAC). (Fuente: DeCS BIREME)

\section{ABSTRACT}

The objective of this case report is to assess the use of autologous bone graft and platelet-rich fibrin (FRP), as guided bone regeneration, after the extraction of a retained canine. A 42-year-old female patient, with a history of controlled hypertension, attended the SERODU dental clinic (Ecuador), due to the presence of prominence in the left nasolabial region. A Computerized Axial Tomography (CT) was performed, in which tooth 1.3 included in the maxilla was observed, in a horizontal position, the crown mesially and the root distally. It was decided to carry out the extraction of the retained canine and the placement of regenerative biomaterials based on FRP and an autologous bone graft, obtained from the right maxillary tuberosity. Clinical and radiographic controls were carried out after surgery. Six months later, adequate healing and images compatible with bone regeneration were observed in the intervened area.

Keywords: Impacted tooth; Platelet Rich Fibrin; Bone Regeneration; Computed Tomography; Computerized Axial Tomography. (Source: MeSH NLM)

${ }^{1}$ Facultad de Odontología, Universidad UTE. Ecuador

adontólogo

${ }^{\mathrm{b}}$ Especialista en Cirugía maxilofacial

Correspondencia:

María José Jarrín

Dirección: Calle Bolívar 6-38 y González Suárez

Correo electrónico: majp_1995@outlook.com

Este es un artículo Open Access distribuido bajo la licencia Creative Commons Atribución 4.0 Internacional (CC BY 4.0)

https://creativecommons.org/licenses/by/4.0/deed.es

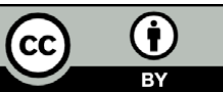

Citar como: Ormaza-Agualsaca C, Lara Analuisa LV, Jarrín-Peñafiel MJ, Castro Pallaroso JA. Exodoncia de canino impactado más regeneración ósea con fibrinarica en plaquetas e injerto de hueso autólogo. Presentación de un caso. KIRU. 2021;18(1):40-47. https://doi.org/10.24265/kiru.2021.v18n1.06 


\section{INTRODUCCIÒN}

Los caninos juegan un papel importante en la apariencia estética, facial, dental, de la misma manera en el desarrollo del arco dental y la oclusión funcional (1). El canino maxilar tiene el periodo de desarrollo más extenso, el área más profunda de desarrollo y un trayecto mucho más complejo para llegar a su posición final (2).

La anomalía en la cual el diente queda incluido en los maxilares y cubierto por su saco pericoronario, más allá de su tiempo de erupción normal ya sea por hueso o por dientes contiguos y existe evidencia clínica y radiográfica de que no habrá lugar en los maxilares se denomina impactación de los caninos ${ }^{(3)}$.

Presenta una prevalencia entre 0,8 y $2,8 \%{ }^{(4)}$. La incidencia se cifra en el $2,4 \%$ en maxilar $(1,4 \%$ derecho y $1 \%$ izquierdo) ${ }^{(5)}$, el $56 \%$ entre 20 y 25 años ${ }^{(6)}$ Prevalece en el sexo femenino ${ }^{(7)}$.

En los últimos 30 años para la corrección de la deficiencia alveolar se ha manejado los injertos óseos ${ }^{(8)}$.

Las opciones de injerto óseo incluyen hueso autógeno, alogénico, xenogénico, sintético y una combinación de los anteriores (9). Los injertos óseos autógenos se consideran "el estándar de oro" debido a su compatibilidad y potenciales osteogénicos para crear el hueso nuevo mediante 3 procesos muy importantes: osteogénesis, osteoinducción y osteoconducción (10).

En la práctica clínica, un injerto óseo de tuberosidad maxilar a menudo se ha utilizado como un injerto particulado para el aumento alveolar deficiente 0 del seno maxilar antes 0 simultáneamente (11).

La Fibrina Rica en Plaquetas (FRP), es un material biocompatible autógeno y concentrado plaquetario de segunda generación (12), contiene leucocitos, plaquetas y factores de crecimiento, indispensables para la cicatrización (13).

En el caso clínico reportado, se observó una imagen radiolúcida compatible con la pieza 13 incluida en el maxilar, posición horizontal de la pieza dental, hacia mesial la corona y la raíz hacia distal con formación completa, por lo que se decidió llevar acabo la cirugía de canino impactado más regeneración ósea guiada con Fibrina rica en plaquetas e injerto de hueso autólogo obtenido de la tuberosidad maxilar derecha. El objetivo de este reporte es valorar la utilidad del injerto autólogo más fibrina rica en plaquetas como regeneración ósea guiada en la extracción de canino retenido.

\section{REPORTE DEL CASO}

Paciente femenino refiere que hace 5 meses aproximadamente presenta una prominencia en la región nasogeniana del lado izquierdo, intraoral se presenta expansión y adelgazamiento de la tabla vestibular.

Exámenes complementarios. Radiografía periapical (Figura 1A), Radiografia panorámica (Figura 1B). Tomografía Axial Computarizada (Figura 2B). Tomografía Computada Cone Beam y exámenes de laboratorio.

Diagnóstico. Canino retenido

Plan de tratamiento. Cirugía de canino retenido más regeneración ósea guiada con FRP e injerto de hueso autólogo.

Aplicación-evolución del plan de tratamiento. Aplicación al paciente de la profilaxis dental y antibiótica (amoxicilina de $500 \mathrm{mg}$ más ácido clavulánico de $125 \mathrm{mg}$ ) 1 hora antes de la cirugía.

Se preparó la mesa quirúrgica, aplicamos la asepsia y antisepsia del área quirúrgica. Se colocaron los campos quirúrgicos (Figura 2A). Se inició el bloqueo anestésico del nervio infraorbitario con técnica extraoral (Figura 2B), del troncular de nervio nasopalatino anterior (Figura 2C), y anestesia a nivel del fondo de surco de la pieza 1.6 con técnica infiltrativa y en la región palatina de la zona mencionada. (Figura 2D), Se utilizó lidocaína al 2\% + epinefrina 1:100 000 . 


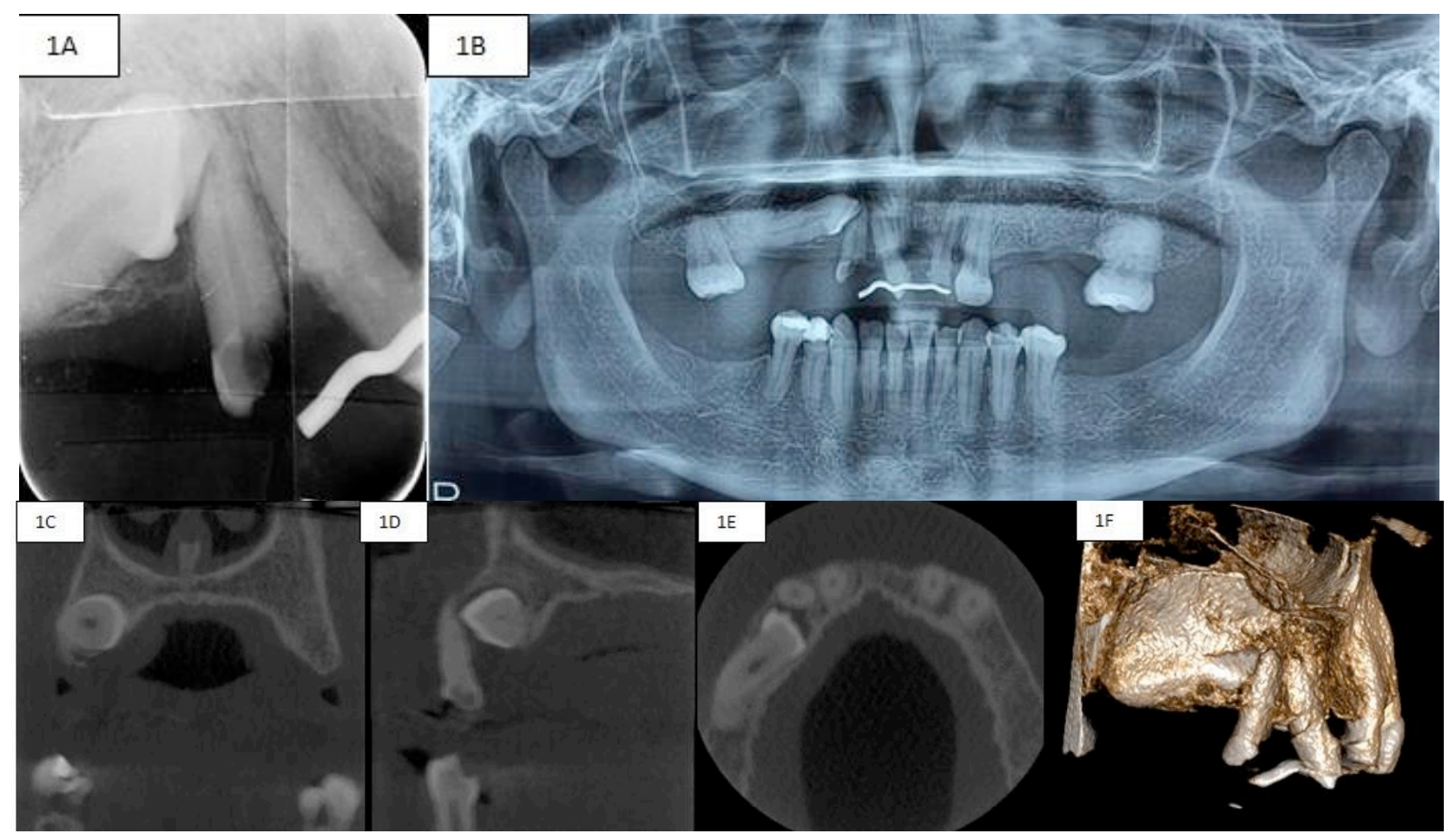

Figura 1A. Radiografía Periapical. 1B. Radiografía Panorámica. 1C. Tomografía Axial Computarizada (corte coronal). 1D. Tomografía Axial Computarizada (Corte sagital). 1E. Tomografía Axial Computarizada (corte axial). 1F.Tomografía Axial Computarizada (Corte 3D)

La incisión se realizó con hoja de bisturí $\mathrm{N}^{\circ} 15$ y un mango $\mathrm{N}^{\circ} 3$, el corte fue profundo hasta llegar al tejido óseo limitando con el periostio (Figura 2E). Seguimos con la elevación del colgajo completo semi Newman con la ayuda de la legra (Figura 2F). Luego se procedió con la osteotomía y ostectomía con la fresa quirúrgica de baja velocidad \#708 más irrigación con solución salina (Figura 2G). Se completó la enucleación de la lesión apical (Figura $2 \mathrm{H}$ ) y extracción de la pieza 1.2 (Figura $2 \mathrm{l}$ ). y del canino retenido (Figura 2J). Posteriormente se realiza el abordaje lineal sulcular a nivel de tuberosidad derecha para obtención de hueso autólogo (Figura 2K). Usando solución fisiológica se efectuó el lavado de la cavidad con la finalidad de eliminar restos de tejido y tener una zona lo mas aséptica posible. Se colocó el hueso autólogo en el defecto óseo del canino (Figura 2L) con recubrimiento de fibrina rico en plaquetas (Figura $2 \mathrm{M}$ ), y se reposicionó el colgajo (Figura $2 \mathrm{~N}$ ). Se realizó la síntesis del colgajo vestibular con liberación de periostio. Se colocaron puntos continuos y separados (Figura 20). Realizada la sutura, ubicamos gasa estéril pidiendo al paciente que ocluyera para evitar hemorragia. Luego procedimos a entregar por escrito una receta médica a la paciente con las indicaciones postoperatorias respectivas.

Seguimiento del tratamiento ejecutado. Las suturas se retiraron a los 8 días de realizada la cirugía . Se observó clínicamente una evolución excelente del proceso de cicatrización.

Evaluación Postoperatoria al mes. La paciente no refirió sintomatología. Se procedió a tomar la radiografía periapical de la zona tratada en la cual se observó una zona radiopaca evidenciando así la regeneración ósea.

Evaluación Postoperatoria a los 6 meses. Nuevamente se tomo una radiografía de la zona tratada en la cual se observa una zona radiopaca compatible con regeneración ósea completa. 


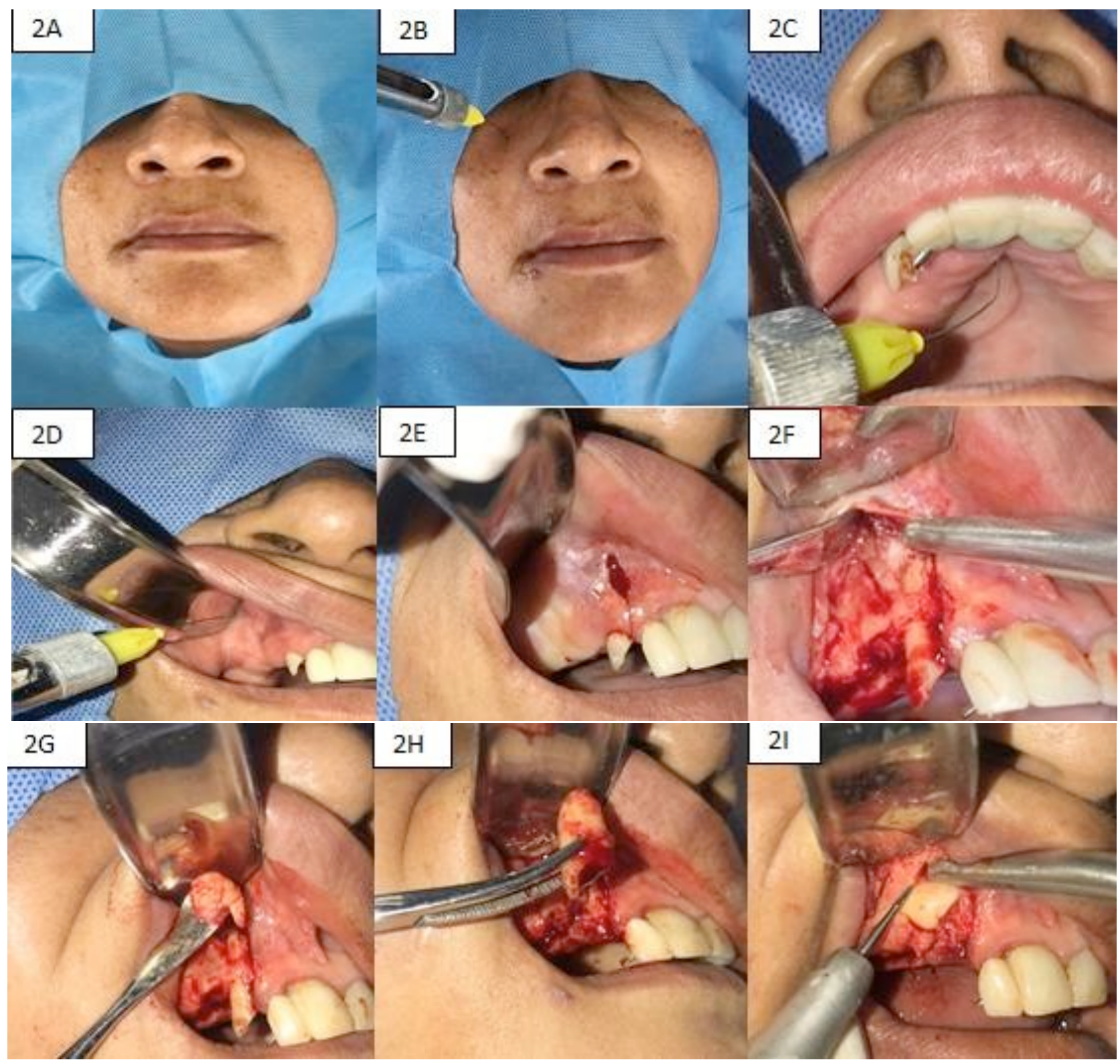

Figura 2A. Colocación de campos quirúrgicos al paciente. 2B Bloqueo anestésico extraoral del nervio infraorbitario. 2C. Bloqueo anestésico del nervio nasopalatino anterior. 2D. Bloqueo anestésico a nivel de fondo de surco de la pieza 16 con técnica infiltrativa. 2E. Incisión semi Newman. 2F. Elevación de colgajo. 2G. Enucleaciòn del proceso periapical. 2H. Avulsión de la pieza N ${ }^{\circ} 1.2$. 21. Osteotomía y ostectomía. 


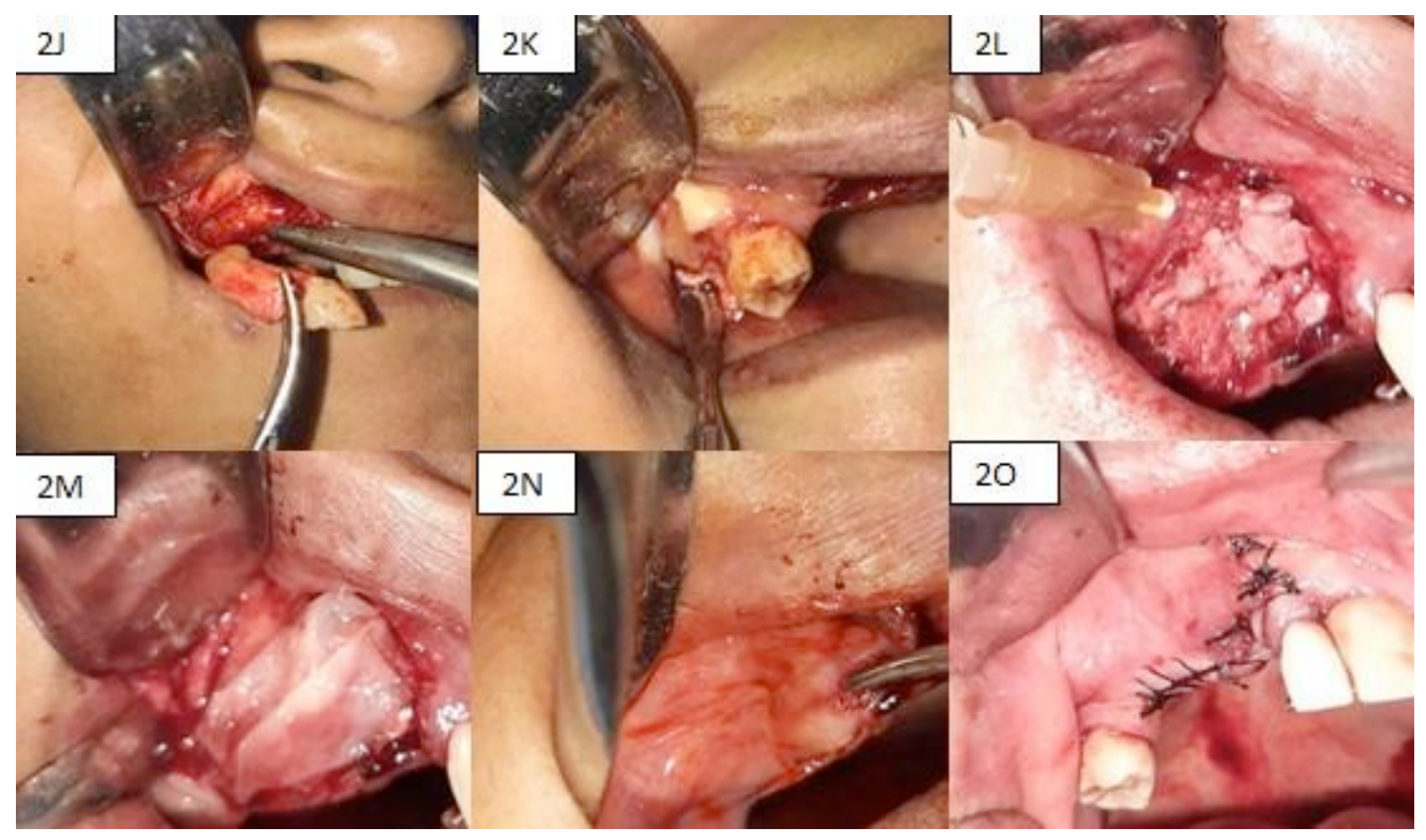

Figura 2J. Luxación y avulsión del canino. 2K. Abordaje lineal sulcular a nivel de tuberosidad derecha. 2L. Colocación de hueso autólogo. 2M. Colocación de membrana de FRP. 2N. Reposición de colgajo. 20. Síntesis de colgajo con puntos continuos y separados.

\section{DISCUSIÓN}

En el presente caso se pudo observar la retención del canino superior. La frecuencia de retención del canino superior es del $34 \%$, tras el tercer molar inferior que se encuentra en el primer lugar ${ }^{(14)}$.

En un estudio realizado por Troya Borges, en un total de 100 entidades patológicas con caninos retenidos, manifestó que la extracción quirúrgica de estos dientes se realiza siempre y cuando no se pueda llevar a cabo una tracción ortodóncica, o cuando coexista alguna patología asociada a la inclusión (quistes voluminosos, infección, rizólisis, necrosis pulpar) o una enfermedad sistémica grave ${ }^{(15)}$.

Los injertos autólogos pueden contener hueso cortical, esponjoso, cortico-esponjoso o bien presentarse en forma de bloque ${ }^{(16)}$. Los injertos corticales presentan una menor cantidad de células osteogénicas, por lo que podrían no ser capaces de mantener la viabilidad celular (17); por el contrario, los injertos esponjosos presentan en principio un mayor tiempo de duración debido a la mayor difusión de nutrientes y grado de revascularización desde el lecho receptor (18).

En un informe reciente, se reportó que la tuberosidad maxilar humana es una fuente adecuada de células osteoprogenitoras para la formación del tejido óseo (19).De otro lado, la calidad de hueso en el maxilar posterior, especialmente en la zona de la tuberosidad del maxilar, generalmente es deficiente (tipo 4), tejido adiposo y una pequeña cantidad de células osteogénicas vitales (20). A pesar de esto, la tuberosidad maxilar es sitio donante de partículas de hueso (21), se usa comúnmente para correcciones relacionadas con el implante de hueso en zonas atróficas y defectos óseos (22). 
La Regeneración Ósea Guiada se utiliza de manera exitosa para tratar diversos tipos de defectos óseos a través del uso de membranas con funciones de barrera, como las membranas de colágeno ${ }^{(23)}$ y en los últimos años se prueban otros métodos con mejores resultados clínicos, como la Fibrina rica en plaquetas (FRP) (24).

Se ha descrito la utilización de membranas de FRP en alvéolos pos-extracción o pos-avulsión siendo el material con la única capacidad de preservación alveolar ${ }^{25)}$, induciendo la formación de hueso tras 6 semanas sin signos de reabsorción ósea (26), se indica usar FRP en combinación con sustitutos óseos para una mejor conexión biológico entre las partículas óseas ${ }^{(27)}$.

La regeneración ósea guiada con fibrina rica en plaquetas (FRP) más injerto autólogo constituye una técnica de tratamiento efectivo, en defectos óseos.

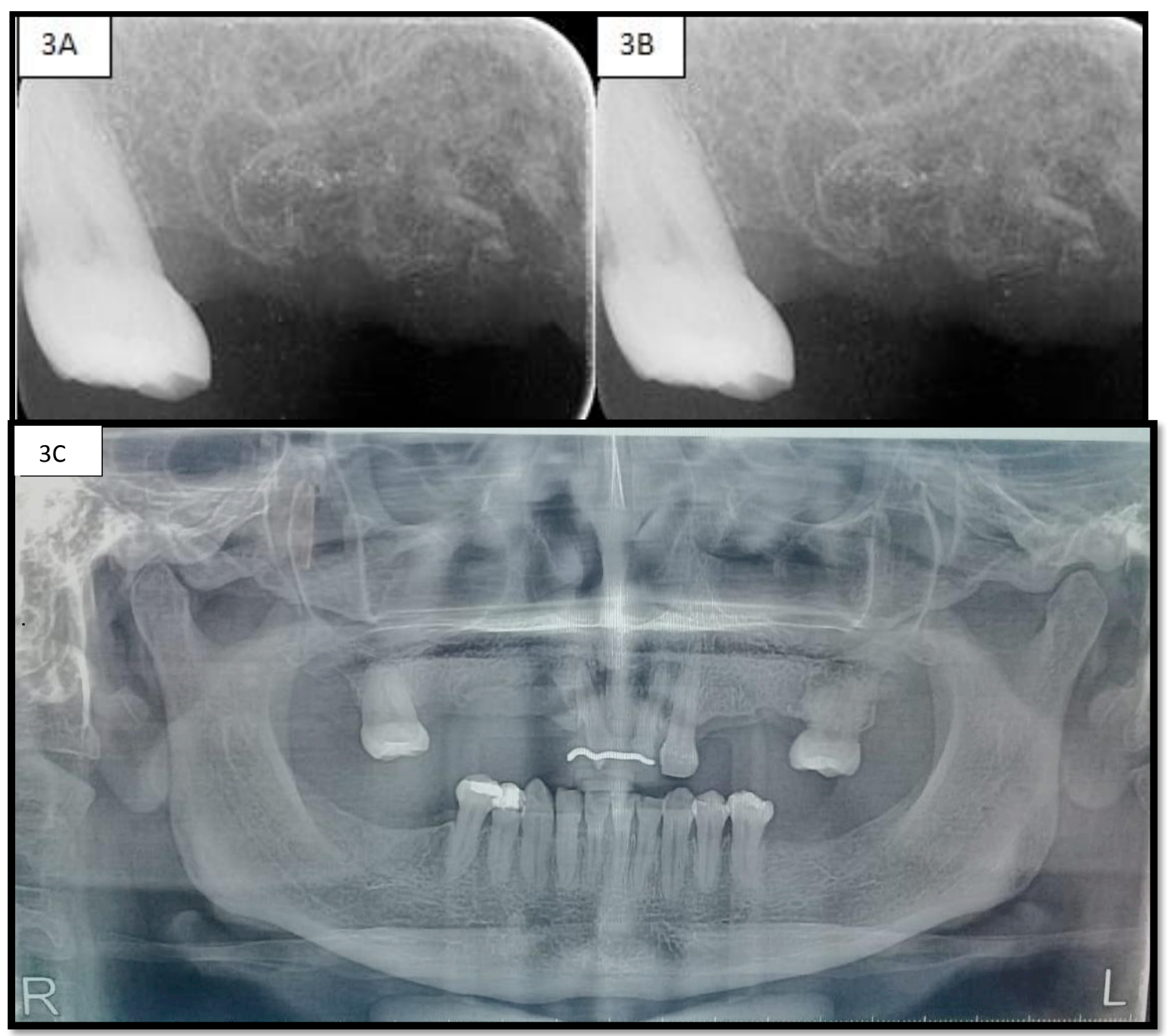

Figura 3A. Radiografía postoperatoria. 3B. Radiografía postoperatorios al mes. 3C. Radiografía postoperatorios a los 6 meses. 
Fuente de financiamiento: El presente caso fue financiado por los autores.

Conflicto de intereses: Los autores declararon no tener conflictos de interés.

\section{REFERENCIAS BIBLIOGRÁFICAS}

1. Chapokas AR, Almas K, Schincaglia GP. The impacted maxillary canine: a proposed classification for surgical exposure. Oral Surg Oral Med Oral Pathol Oral Radiol. 2012;113(2):222-228.

2. Becker A, Chaushu S. Etiology of maxillary canine impaction: a review. Am J Orthod Dentofacial Orthop. 2015;148(4):557-567.

3. Ucar FI, Celebi AA, Tan E, Topcuoğlu T, Sekerci AE. Effects of impacted maxillary canines on root resorption of lateral incisors : A cone beam computed tomography study. Auswirkungen impaktierter Oberkiefereckzähne auf eine Wurzelresorption im Bereich der lateralen Inzisivi : Eine Studie mit digitaler Volumentomograpie. J Orofac Orthop. 2017;78(3):233-240.

4. Sarica I, Derindag G, Kurtuldu E, Naralan ME, Caglayan F. A retrospective study: Do all impacted teeth cause pathology?. Niger J Clin Pract. 2019;22(4):527-533.

5. Cacciatore G, Poletti L, Sforza C. Early diagnosed impacted maxillary canines and the morphology of the maxilla: a three-dimensional study. Prog Orthod. [Internet].2018[cited 2020 July 05];4(1):32-34. Available in: https://www.ncbi.nlm.nih.gov/pubmed/ 3000934 0

6. An, S., Wang, J., Li, J., Cheng, Q., Jiang, C. M., Wang, Y. T., Huang, Y. F., Yu, W. J., Gou, Y. C., \& Xiao, L.. Comparison of methods for localization of impacted maxillary canines by panoramic radiographs. Dento maxillo facial radiology. 2013; 42(8).

7. Al-Zoubi, H., Alharbi, A. A., Ferguson, D. J., \& Zafar, M. S. (2017). Frequency of impacted teeth and categorization of impacted canines: A retrospective radiographic study using orthopantomograms. European journal of dentistry. 2017; 11(1): 117-121.

8. Sakkas A, Wilde F, Heufelder M, Winter $K$, Schramm A. Autogenous bone grafts in oral implantology-is it still a "gold standard"? A consecutive review of 279 patients with 456 clinical procedures. Int $\mathrm{J}$ Implant Dent. 2017;3(1):23.

9. Calvo-Guirado JL, Aguilar-Salvatierra A, Ramírez-Fernández MP, Maté Sánchez de Val JE, Delgado-Ruíz RA, Gómez-Moreno G. Bone response to collagenized xenografts of porcine origin, $\mathrm{Mp} 3(\AA)$, and a bovine bone mineral grafting (4Bone(TM)) XBM) grafts in tibia defects: experimental study in rabbits. Clin Oral Implants Res 2016; 27:1039-46.

10. Danesh-Sani SA, Tarnow D, Yip JK, Mojaver R. The influence of cortical bone perforation on guided bone regeneration in humans. Int $\mathrm{J}$ Oral Maxillofac Surg.2017; 46:261-266.

11. Salgado-Peralvo, Ángel Orión, Salgado-García, Ángel, \& Arriba-Fuente, Lorenzo. Nuevas tendencias en regeneración tisular: fibrina rica en plaquetas y leucocitos. Revista Española de Cirugía Oral y Maxilofacial.2017; 39(2):91-98.

12. Dohan DM, Bielecki T, Jimbo R, et al. Do the fibrin architecture and leukocyte content influence the growth factor release of platelet concentrates? An evidence-based answer comparing a pure platelet-rich plasma (P-PRP) gel and a leukocyte- and platelet-rich fibrin ( $\mathrm{L}-$ PRF). Curr Pharm Biotechnol. 2012;13(7):1145-1152.

13. Belkhede, S. G., Salaria, S. K., \& Aggarwal, R. Comparative evaluation of the platelet-rich fibrin bandage versus gelatin sponge-assisted palatal wound healing of free gingival graft donor site: A case series. Journal of Indian Society of Periodontology.2019;23(6), 589-592.

14. Alqerban, A., Jacobs, R., Fieuws, S., \& Willems, G. Predictors of root resorption associated with maxillary canine impaction in panoramic images. European journal of orthodontics. . [Internet].2016[cited 2020 July 05];38(3):292-299. Available in: https://www.ncbi.nlm.nih.gov/pubmed/ 26150321

15. Troya Borges E, Martínez Abreu J, Padilla Suárez $\mathrm{E}$, Matos Campos $\mathrm{M}$. Tratamiento quirúrgico de caninos retenidos en el municipio Colón. Años $2013 \quad$ - 2014.. Revmedicaelectronica [Internet]. 2016 [citado 2020 July 05];38(2).Available in en: http://www.revmedicaelectronica.sld.cu/index.p $\mathrm{hp} / \mathrm{rme} / \mathrm{article} / \mathrm{view} / 1608$

16. R.M. Baiju, R. A. Autologous platelet-rich fibrin: A boon to periodontal regeneration. Report of two different clinical applications. Health Sciences. 2013; 2: 1-13.

17. Sheikh, Z., Hamdan, N., Ikeda, Y., Grynpas, M., Ganss, B., \& Glogauer, M. Natural graft tissues and synthetic biomaterials for periodontal and alveolar bone reconstructive applications: a review. Biomaterials research.2017; 21(9).

18. Titsinides, S., Agrogiannis, G. y Karatzas, T. (2019). Materiales de injerto óseo en la reconstrucción dentoalveolar: una revisión exhaustiva. The Japanese dental science review.2019; 55 (1):26-32.

19. Younes, R. y Khairallah, CM. El injerto de tuberosidad autólogo "One Piece": un concepto contemporáneo en la preservación de crestas. Informes de casos en odontología.2020. 
20. Salmón, B., Liu, B., Shen, E., Chen, T., Li, J., Gillette, M., Ransom, RC, Ezran, M., Johnson, CA, Castillo, AB, Shen, WJ , Kraemer, FB, Smith, AA y Helms, JA. Los injertos óseos activados por WNT reparan lesiones osteonecróticas en animales de edad avanzada. Informes científicos.2017; 7(1):14254.

21. Stumbras, A., Kuliesius, P., Januzis, G., \& Juodzbalys, G. Alveolar Ridge Preservation after Tooth Extraction Using Different Bone Graft Materials and Autologous Platelet Concentrates: a Systematic Review. Journal of oral \& maxillofacial research.2019;10(1).

22. tolstunov.dds, L.. Maxillary tuberosity block bone graft: innovate technique and case report. J Oral Maxillofac Surg.2009; 67:1723-1729.

23. Strauss, F. J., Stähli, A., \& Gruber, R. The use of platelet-rich fibrin to enhance the outcomes of implant therapy: A systematic review. Clinical oral implants research.2018; 29 (18): 6-19.
24. Albanese, A., Licata, ME, Polizzi, B. y Campisi, G. Plasma rico en plaquetas (PRP) en cirugía dental y oral: desde la cicatrización de heridas hasta la regeneración ósea. Inmunidad y envejecimiento: I y A. 2013; 10 (1): 23.

25. Endod, O. S. Comparison of effects of PRF dressing in wound healing of palatal donor site Turing free gingival grafting procedures with no dressing at the donor site. J Res Adv Dent.2015; 4:69-74

26. JeM. Del Corso, A. V. Current knowledge and perspectives for the use of platelet-rich plasma (PRP) and platelet-rich fibrin (PRF) in oral and maxillofacial surgery. Part I: Periodontal and dentoalveolar surgery. Curr Pharm Biotechnol.2012; 13(1): 1207-1230.

27. Miron RJ, Fujioka-Kobayashi M, Hernandez $M$, et al. Injectable platelet rich fibrin (i-PRF): opportunities in regenerative dentistry?. Clin Oral Investig. 2017;21(8):2619-2627.

Cinthya Geovanna Ormaza Agualsaca

ORCID iD: (b) http://orcid.org/ 0000-0003-1820-9876

cinthyaormaza222@gmail.com

Lenin Vladimir Lara Analuisa

ORCID iD: (D) http://orcid.org/000-0002-7361-3102

vladylara12_@hotmail.com

María José Jarrìn Peñafiel

ORCID iD: (b) http://orcid.org/ 0000-0002-1875-1254

majp_1995@outlook.com

Jaime Arturo Castro Pallaroso

ORCID iD: (D) http://orcid.org/ 0000-0002-3477-6953

ja.castro@udlanet.ec

Copyright (C) La revista. La revista Kiru es publicada por la Facultad de Odontología de la Universidad de San Martín de Porres, en Lima, Perú. 